\title{
Las dimensiones sociales de los riesgos en torno a conflictos socioambientales por energía en Chile. La confianza en el conflicto de la Termoeléctrica Castilla
}

\author{
Arturo Vallejos-Romero \\ Universidad de la Frontera. Núcleo de Investigación Científico Tecnológico en Ciencias \\ Sociales y Humanidades, \\ arturo.vallejos@ufrontera.cl
}

\section{Sonia Salvo}

Universidad de la Frontera. Departamento de Matemáticas y Estadísticas, Laboratorio de Investigación en Ciencias Sociales Aplicadas

sonia.salvo@ufrontera.cl

\section{Jaime Garrido}

Universidad de la Frontera. Departamento de Ciencias Sociales

jaime.garrido.c@ufrontera.cl

\section{Resumen}

Los riesgos ambientales relacionados con proyectos termoeléctricos en Chile se han expandido y complejizado en los últimos años, lo cual ha generado conflictos sociales y ha minado la capacidad privada e institucional para prevenirlos, administrarlos y resolverlos. La confianza, entendida como una disposición configurativa de relaciones sociales, aparece como un factor clave para los desafíos de la gobernanza de riesgos y la conflictividad socioambiental. El artículo presenta los resultados de una encuesta, específicamente, el análisis de la dimensión confianza, con el propósito de establecer una relación entre individuos y variables a través de un análisis biplot simétrico. Se distinguieron tres grupos de individuos, lo cual permitió caracterizar un perfil específico para cada caso frente al papel que desempeñaron las instituciones públicas y privadas durante la gestación, el desarrollo y el conflicto que se generó en torno al proyecto Termoeléctrica Castilla en la región de Atacama (Chile). Entre los resultados, destaca que las dimensiones basadas en la competencia, la habilidad y la benevolencia son las que aportan mayor variabilidad para explicar la percepción de confianza en el conflicto Castilla. Por su parte, el nivel de aceptabilidad de la termoeléctrica también está relacionado con la disposición a participar en organizaciones sociales y en acciones colectivas contenciosas.

Palabras clave: riesgos sociales; riesgos ambientales; conflictos sociales; confianza. 
Abstract. Social Dimensions of Risks Relating to Socio-Environmental Conflicts over Energy in Chile: "Trust" in the Castilla Thermoelectric Conflict.

Environmental risks related to thermoelectric projects in Chile have expanded and become more complex in recent years, giving rise to new social conflicts and dismissing the public and private institutional capacity to prevent, administrate and resolve them. Trust, understood as a configurative disposition of social relations, appears as a key factor for the challenges faced by the governance of risks and socio-environmental conflict. This article presents part of the results of a survey, specifically the analysis of the dimension of trust, with the purpose of establishing a relationship between individuals and variables through a symmetric biplot analysis. Three groups of individuals are identified, thus permitting us to characterize a specific profile for each case according to the role played by public and private institutions during the gestation, development, and conflict that arose around the Castilla Thermoelectric project in the Atacama Region of Chile. We highlight that the competence-ability and benevolent dimensions offer a greater variability for explaining the perception of trust in the Castilla conflict. In addition, the level of acceptability of the thermoelectric project is also related to the disposition to participate in social organizations and contentious collective actions.

Keywords: social risks; environmental risks; social conflicts; trust.

\section{Sumario}

1. Los riesgos y la conflictividad socioambiental. El problema

2. La confianza y su relevancia en los conflictos socioambientales

3. El caso de la Termoeléctrica Castilla en la región de Atacama

4. Metodología
5. Resultados

7. Conclusiones

Agradecimientos

Referencias bibliográficas

Anexo 1. Distribuciones de los promedios estandarizados por grupos

\section{Los riesgos y la conflictividad socioambiental. El problema}

Los riesgos en torno a conflictos por infraestructuras energéticas en Chile han sido expresiones y consecuencias del propio modelo de desarrollo y se han convertido, por un lado, en uno de los principales focos y fuentes de tensión en la actualidad y, por otro, en situaciones que ponen en peligro los requisitos básicos para la vida social y natural (Beck, 2006; Giddens, 1993, 1996a, 1996b; Bauman, 2006), lo cual provoca conflictos de compleja solución (Luhmann, 1996b). La teoría del riesgo (Giddens, 1993; Beck, 2002, 2006, 2008) concibe las contingencias y los peligros como globales, imprevisibles e inevitables. Ello resquebraja la idea de seguridad y afecta no solo a modernidades centrales, sino también a periféricas como América Latina, donde sus instituciones, que generan dispositivos para prevenir y controlar los riesgos que producen, se muestran cada vez más ineficientes ante sus manifestaciones. En este contexto, las respuestas sociales ante las proximidades de un daño se vuelven complejas y las responsabilidades tienden a ser difíciles de delimitar. 
En este sentido, su tematización como problemáticas sociales crean una «inseguridad en la sociedad» que activa nuevas formas de responder a los peligros sociales y ambientales (Luhmann, 1992, 1996b, 2007) y ello provoca que la sociedad y sus mecanismos aseguradores se muestren cada vez más limitados en sus formas de reflexión, decisión y acción.

Tras esta breve contextualización, el artículo que presentamos pretende dar cuenta de la relevancia que cobra la confianza (interpersonal e institucional) en la gestión de los riesgos asociados a conflictos socioambientales ligados a la energía. Conceptualizada la confianza como una de las dimensiones sociales de los riesgos socioambientales (Vallejos-Romero et al., 2014), la creemos clave para dar cuenta de la conflictividad relacionada con ellos, así como de la gestión o la gobernanza de los conflictos, en este caso, el conflicto Termoeléctrica Castilla en la región de Atacama, en Chile (de ahora en adelante, "Castilla»).

Los riesgos en torno a conflictos socioambientales, especialmente los ligados a energía y generados por termoeléctricas en Chile, pueden ser observados como resultados del diseño y la implementación de los modelos de desarrollo económico, anclados en pujantes procesos de industrialización, urbanización y grandes megaproyectos que han tenido lugar en las últimas décadas (Altieri y Rojas, 1999; Quiroga, 2001; Ricaldi, 1999). Los problemas y los riesgos socioambientales derivados de este proceso, no solo se han expandido y complejizado en los últimos tiempos (Camus y Hajek, 1998; INAP, 2006), sino que la capacidad privada e institucional para prevenirlos y resolverlos se ha visto sobrepasada (OCDE, 2005; Sepúlveda y Rojas, 2010).

Ante tales conflictos y riesgos, la sociedad ha reaccionado con una conflictividad que ha ido escalando en intensidad (Fiske, 2000; Sabatini y Sepúlveda, 1997; Sabatini 1998), y donde tales reacciones no han sido respuestas automáticas, sino mediatizadas por el significado que los problemas socioambientales tienen para las organizaciones y los actores sociales (Beck, 1995; Rojas, 1995; Sepúlveda y Geisse, 1995). Por un lado, la expresión social ante el aumento de los riesgos y de sus externalidades ha generado conflictos con una gran reverberancia social, mientras que, por otro lado, los problemas socioambientales se multiplican sin que necesariamente generen manifestaciones explícitas, lo que no quiere decir que no sean visibilizados o percibidos por quienes son impactados (Espluga, 2004, 2007; Espluga y Farré, 2007).

Ante el contexto planteado en los párrafos precedentes, el artículo que presentamos pone en evidencia que los riesgos son instancias privilegiadas para observar las reacciones y las respuestas sociales ante las externalidades socioambientales que producen (Vallejos-Romero et al., 2014). Así también, esta perspectiva permite configurar y perfilar de una mejor manera los actores que gatillan y se confrontan en tales fenómenos, así como la relación que se da entre estos, evidenciando y poniendo en tensión, al mismo tiempo, las percepciones y las relaciones de los distintos actores hacia los problemas y los conflictos socioambientales.

El potencial de análisis de los riesgos visibilizados en la producción de energía es clave y se expresa, aunque no únicamente, en las percepciones y con- 
figuraciones de los actores y las relaciones de confianza que se establecen - $\mathrm{u}$ obstaculizan- a partir de las respuestas a los impactos y las externalidades del desarrollo. Los conflictos manifiestos en torno al sector eléctrico en Chile han ido escalando en cantidad e intensidad, en tanto la gestión privada y pública no ha dado cuenta debidamente de ellos, y la literatura (Wynne, 1992, 1996) nos muestra la importancia de aspectos sociales e institucionales como la confianza y que algunos autores han identificado como el foco principal de los desafíos y las limitaciones de la gestión de riesgos (Espluga et al., 2010, 2014).

Explorar la problemática planteada es relevante para la gestión de los riesgos, pues las instancias públicas en Chile deberán tomar decisiones en torno a su matriz energética, donde la principal fuerza de esta estará en la fuente hidráulica (agua) y termoeléctrica (carbón y petróleo) (Aguilar y Valenzuela, 2010; VVAA, 2010). La tensión social que esta situación está generando y probablemente generará será profunda, con una expansión potencial de los problemas y los conflictos en torno a la producción de energía, donde las dimensiones sociales como la confianza son importantes para la gestión y su análisis vital para generar conocimiento en torno a un incipiente campo interdisciplinario en Chile.

Planteada la relevancia del tema, así como el problema que emerge tanto para la intervención pública como para el avance en el conocimiento científico, el artículo tiene como objetivo dar cuenta de las relaciones y percepciones de confianza de los actores sociales sobre las instituciones públicas y privadas. Nos interrogamos: ¿cómo y de qué tipo son las relaciones de confianza que emanan de los actores sociales hacia las instituciones públicas y privadas? ¿Quiénes son aquellos actores sociales o cuáles son sus características? ¿Cómo evalúan estos actores a las instituciones encargadas de gestionar los riesgos en torno a conflictos como el de la Termoeléctrica Castilla? ¿De qué tipo es la confianza de los actores hacia las instituciones públicas y privadas? ¿Qué diferentes grados de confianza hay entre los diversos actores sociales? El análisis de un conflicto socioambiental concreto, como el de la Termoeléctrica Castilla, nos permitirá ilustrar la complejidad subyacente a los riesgos tecnológicos, mediante la identificación y la caracterización de los grupos de actores en conflicto.

\section{La confianza y su relevancia en los conflictos socioambientales}

La confianza puede ser entendida, en términos generales, como un dispositivo que ordena y reduce la complejidad de la convivencia social, por lo tanto, es un recurso para cualquier acción de futuro que probabiliza los comportamientos y garantiza cierta seguridad. Cuando este sustento se pierde, tienden a cuestionarse las acciones y las capacidades institucionales por parte de la sociedad, de tal modo que todo diseño propiciado por aquellas instituciones (públicas y privadas) se encuentra con dificultades para dar cuenta de los riesgos que genera la dinámica social, que pueden traducirse en conflictos socioambientales como los termoeléctricos en Chile, entre otros. 
En este escenario, los estudios del riesgo han comenzado a prestar una fuerte atención a la confianza, que aparece como «un prerrequisito para conseguir una efectiva comunicación y gobernanza del riesgo» (Kasperson et al., 1992, citado por Espluga et al., 2009: 3). Para el escrito que presentamos, la confianza es un mecanismo de reducción de complejidad (Luhmann, 1996a), una dimensión que, junto a otras, permite que la sociedad sea posible.

Siendo la confianza un reductor de complejidad social, se transforma en sustento ante una colectividad que no puede prometer seguridades ni certidumbres, de modo que actúa como un dispositivo que aumenta la probabilidad de las instituciones y del público para obrar coherentemente en un entorno altamente complejo y desbordante. Ello posibilita tener mayores probabilidades de certidumbre ante el futuro. Ante la complejidad del entorno y la información parcial que se pueda tener, la confianza establece un cierto grado de certeza interna que garantiza cada una de las operaciones en el momento de tomar decisiones.

En el marco planteado, argumentamos que la confianza no elimina los riesgos, sino que los hace más aceptables y, ante lo que no controlamos, esta nos ayuda a soportar situaciones aventuradas y peligrosas a las que nos expone la sociedad. En otras palabras, confiar es poder soportar la elevada incertidumbre ante el futuro. Así, la confianza haría posible la estabilidad social y ayudaría a dar cuenta de los riesgos en una sociedad que se vuelve cada vez más contingente y donde cada decisión tiene más probabilidades de ser decepcionada. En síntesis, la confianza sería una especie de impronta o sello social que estaría en las personas e instituciones y que posibilitaría la dinámica social.

Espluga et al. (2009), Rodríguez y Majluf (2003), Majluf et al. (2003) y Rodríguez (2009) exponen profusamente los esfuerzos particulares de conceptualizar la confianza pública desde la sociología, la psicología y las ciencias políticas. Rodríguez, Majluf, Abarca y Mingo elaboran un modelo para medir la confianza interpersonal, donde proponen una confianza basada en la razón y en el afecto, y valoran la confianza directa y la indirecta. En la primera (razón), los autores mencionan componentes como la competencia o la habilidad, la benevolencia y la integridad, el sentido de justicia, el cumplimiento de compromisos y la consistencia del comportamiento, mientras que, en la segunda (afecto), identifican la apertura comunicacional y la ausencia de conflicto emocional.

Esta propuesta nos permite ordenar las dimensiones técnicas y afectivas que proponen Espluga et al. (2009) y nos ayuda, más allá de la variable, a poner el acento en un marco orientador que dé cuenta de las pretensiones de nuestro trabajo. Más específicamente, y en un amplio recorrido por la bibliografía especializada, Espluga et al. (2009) enmarcan la confianza como un constructo multidimensional, donde hay aspectos técnicos como la competencia, la capacidad, la eficiencia, la consistencia o el conocimiento y la experiencia experta; mientras que, en la dimensión afectiva, se encuentran elementos como la objetividad o la imparcialidad, la honradez, la buena voluntad, la preocupación por la salud y el bien común, la integridad y el compromiso con los intereses de los demás. 
La producción teórica conceptual ha observado la confianza desde dos vertientes: una moral y otra estratégica (Bulloch, 2013). Nuestra aproximación se aparta de la primera vertiente, que concibe la confianza como una creencia en la bondad de la naturaleza humana en general (Yamagishi y Yamagishi, 1994, citado por Bulloch, 2013), y más bien se hace cargo de una visión estratégica de confianza donde los individuos confían porque es razonable hacerlo; en la cual la confianza será posible si tiene siempre algún depositario y donde la información y la experiencia son vitales. Haciéndonos cargo de una confianza estratégica, por un lado, esta se torna relevante en la sociedad actual, pues actúa como cemento social que ha ganado regularidad y se ha hecho necesaria para accionar diariamente sin preguntarnos o cuestionarnos sobre las formas que la sociedad ha estabilizado, que, si así fuera, nos llevaría a una hipertrofia que haría poco probable producir y reproducir lo social. Por otro lado, la confianza es una reductora de la complejidad social y puede ser observada, testeada y medida a través de variables e indicadores que puedan dar cuenta de sus rendimientos en una sociedad moderna, especialmente - para nuestro caso- en conflictos socioambientales en torno a la cuestión de la energía en Chile.

En este sentido, apartándonos de una mirada moral, normativa o de control, es relevante indagar en el perfil y en las relaciones de confianza entre los actores implicados en conflictos socioambientales como el de la Termoeléctrica Castilla. En lo operativo, resulta necesario observar dimensiones objetivas y subjetivas de la confianza en las instituciones y en las relaciones interpersonales como las que se presentan en la tabla 1 .

Tabla 1. Dimensiones generales y específicas para la percepción de confianza

\begin{tabular}{|c|c|c|}
\hline \multirow[b]{2}{*}{ Dimensiones generales } & \multicolumn{2}{|c|}{ Dimensiones específicas } \\
\hline & Competencia técnica & Afectiva \\
\hline Competencia y habilidad & $\begin{array}{l}\text { - Experiencia (qué han realizado). } \\
\text { - Eficiencia (cómo lo han realizado). } \\
\text { - Capacidades (recursos finan- } \\
\text { cieros, técnicos, humanos e } \\
\text { infraestructura). } \\
\text { —Competencias (técnicas como } \\
\text { legales). }\end{array}$ & \\
\hline Benevolencia & & $\begin{array}{l}\text { - Preocupación por la salud } \\
\text { y el bien común. } \\
\text {-Buena voluntad. }\end{array}$ \\
\hline Integridad & & $\begin{array}{l}\text { - Honradez. } \\
\text { - Transparencia. }\end{array}$ \\
\hline Sentido de justicia & & —Objetividad e imparcialidad. \\
\hline $\begin{array}{l}\text { Cumplimiento } \\
\text { de compromisos }\end{array}$ & & $\begin{array}{l}\text { - Compromiso con los } \\
\text { intereses de los demás. }\end{array}$ \\
\hline $\begin{array}{l}\text { Consistencia } \\
\text { comportamiento }\end{array}$ & $\begin{array}{l}\text { - Consistencia y predictibilidad de } \\
\text { comportamientos y } \\
\text { argumentos. }\end{array}$ & \\
\hline
\end{tabular}

Fuente: adaptado de Rodríguez y Majluf (2003) y Espluga et al. (2009). 
La tabla 1 nos ofrece la posibilidad de visibilizar y perfilar las posibles relaciones de confianza de los actores implicados (instituciones y ciudadanos) en torno al conflicto socioambiental Castilla en la región de Atacama (Chile), vital para dar cuenta del objetivo que persigue nuestro escrito.

\section{El caso de la Termoeléctrica Castilla en la región de Atacama}

El proyecto de la Termoeléctrica Castilla (2008-2010) pretendía instalar una central termoeléctrica a petróleo diesel y una central termoeléctrica a carbón, que, en conjunto, constituirían una de las mayores centrales de producción de energía en Chile y la mayor central a carbón en Sudamérica. Los montos de inversión rondaban los UM\$4.400, un presupuesto de los más altos del país en este tipo de operaciones. Se trataba de un megaproyecto industrial con grandes impactos ambientales y sociales.

La propuesta de instalación generó una alta contestación social, tanto a nivel nacional como internacional, que desencadenó un conflicto que enfrentó a distintos actores de la comunidad local y nacional. Por un lado, se articularon las posiciones de apoyo, que señalaron que el proyecto era necesario teniendo en cuenta «que las energías limpias solo pueden cubrir una parte de la demanda y el resto del abastecimiento deberá ser siempre desarrollado por energías hidroeléctricas y térmicas» (Vilches, 2010). De acuerdo con la normativa local, la propuesta estaba resguardada, dado que el plan regulador definía un uso industrial para la zona. Por otro lado, los opositores al proyecto indicaban y argumentaban los elevados daños que ocasionaría al ecosistema de la zona, aduciendo «[...] que para poder enfriar los generadores, se sacará agua del mar, que será devuelta con aproximadamente $8^{\circ} \mathrm{C}$ más, lo que provocaría la muerte de peces y mariscos del sector, además de dejar sin trabajo a muchas familias de pescadores de la zona» (Giro País, 2011).

Siendo el carbón un producto contaminante y tóxico, con altas externalidades negativas para el medio natural y humano, el proyecto pone en tensión el desarrollo de la zona, los ejes de la matriz energética, las decisiones que se toman para su implementación (altamente cuestionadas), con serios impactos potenciales en la calidad de vida y en la salud de las comunidades, así como el impacto en las actividades de los pescadores artesanales y el patrimonio del Totoral (zona de pescadores artesanales ubicada en la Tercera Región de Atacama).

\section{Metodología}

Se aplicó una encuesta a la población residente en la zona de conflicto que incluyó las comunas de Caldera y Copiapó, en la región de Atacama, al norte de Chile ${ }^{1}$.

1. Proyecto Fondecyt Regular 1120554, financiado por el Fondo Nacional de Ciencia y Tecnología (Fondecyt) de Chile. Dicho instrumento es uno de los utilizados por la investigación Las dimensiones sociales. Confianza y comunicación de los riesgos ecológicos en conflictos socioambientales por energía en Chile: Los casos de Castilla e HidroAysén, entre las que se contaban también técnicas dialógicas a actores relevantes de la comunidad, instituciones públicas y empresas privadas (entrevistas y grupos de discusión). Aquí solo nos centraremos en los resultados de la encuesta por cuestionario. 
En términos generales, el instrumento se construyó en base a cinco dimensiones. Las tres primeras se orientaron en función del perfil del ciudadano, cuya finalidad era distinguir sus acciones y disposiciones en torno a los problemas socioambientales. Estas dimensiones son: identificación y caracterización (nivel educacional, estado conyugal, situación ocupacional, pertenencia religiosa, pueblos originarios); percepción e inclusión social (capital social, cultura cívica, identidad territorial) y percepción de riesgo socioambiental (información y aceptabilidad). Las dos últimas respondían al propósito principal de la investigación: confianza (objetiva y subjetiva) y comunicación de riesgos (técnica y reflexiva). Para cada dimensión, se formularon varias preguntas o afirmaciones a las que las personas encuestadas debían responder según su grado de acuerdo o desacuerdo en una escala de 1 a 5 . Específicamente, y teniendo como foco los resultados de la investigación que presentamos, se toman los datos, los análisis y los resultados de la dimensión confianza. Las demás son mencionadas como partes constituyentes del instrumento que se enmarca en una investigación mayor.

El marco muestral de la encuesta tomó como universo el número total de viviendas del área urbana para las comunas señaladas, lo que equivale a 39.815 viviendas distribuidas en 33 zonas censales de acuerdo con el Censo Nacional de Población y Vivienda del año 2002. Para determinar la muestra, se utilizó un muestreo aleatorio estratificado y dicotómico (Scheaffer et al., 1987). El tamaño de la muestra para cada uno de los estratos (zonas censales) se obtuvo con un nivel de confianza del $95 \%$, un error de estimación del 5\% y una varianza máxima de 0,25 (considerando $p=q=0,5$ ). Con estas consideraciones, se obtuvo una muestra final de 309 viviendas, distribuidas proporcionalmente en cada una de las zonas censales, entre las que se encuestaron a 580 personas.

El análisis de los datos se hizo mediante el biplot de Gabriel, técnica estadística que posibilita representar de manera simultánea dos ejes, individuos y variables, lo cual permite caracterizar a los entrevistados y situar la confianza como una variable relevante para observar las tensiones en la instalación de proyectos energéticos como los aquí analizados. Para el análisis descriptivo de datos, se utilizó el programa JMP (versión 10, SAS Institute Inc.) y para el análisis de clúster basado en los factores obtenidos por el biplot simétrico, el programa PMD Biplots para Minería de Datos (un sistema de minería de datos basado en biplots) (Vairinhos, 2003). Todas las variables del instrumento que resultaron relevantes se estandarizaron (media cero y desviación típica igual a uno) para hacerlas comparables. Así, una variable estandarizada con valores por debajo de cero significa que los individuos optaron mayoritariamente por evaluaciones negativas, y si están sobre el promedio, lo contrario.

Para vincular el perfil de los ciudadanos con la dimensión y las variables que componen la confianza, se realizó un análisis de clúster jerárquico basado en las dimensiones latentes obtenidas a través de un análisis biplot simétrico de Gabriel, técnica que permite determinar las variables responsables de las agrupaciones obtenidas. Específicamente, esta técnica de análisis multivariado 
de datos permite ordenar gráficamente en un plano las interrelaciones entre individuos y variables, las cuales se analizan a través de las proyecciones de los puntos que representan a los individuos sobre los vectores que representan a las variables, lo que posibilita determinar cuáles son las que más diferencian a subconjuntos de individuos, es decir, la construcción de perfiles.

Para la interpretación del plano factorial, es importante considerar que las distancias entre los marcadores fila se interpretan como una función inversa de sus similitudes, de tal forma que los marcadores próximos (individuos) son más semejantes. Esta propiedad permite identificar individuos con perfiles similares. La longitud de los marcadores columna (vectores) aproximan la desviación típica de las variables. Los cosenos de los ángulos entre los vectores columna aproximan las correlaciones entre los indicadores, de modo que ángulos agudos se asocian a indicadores con alta correlación positiva, ángulos obtusos indican correlación negativa y ángulos rectos señalan variables no correlacionadas. El orden de las proyecciones ortogonales de los marcadores fila (puntos) sobre un marcador columna (vector) aproxima el orden de los elementos fila (individuos) en esa columna (la misma propiedad se cumple para la proyección de los marcadores columna en la dirección definida por un marcador fila). Cuanto mayor es la proyección de un punto sobre un vector, más se desvía el centro de la media de ese indicador.

\section{Resultados}

Lo que se presenta en este artículo es la configuración de perfiles de los encuestados en grupos diferenciados en torno al análisis de la confianza, una de las dimensiones centrales de la investigación que permite ilustrar de mejor manera la complejidad subyacente a los riesgos que genera la conflictividad socioambiental.

Mediante el instrumento (encuesta por cuestionario), se preguntó sobre la percepción de confianza en instituciones públicas y privadas como el Municipio (M), el Gobierno Regional (GR), el Ministerio del Medio Ambiente (MMA), Castilla Empresa (E), la Secretaría Regional Ministerial de Salud (Ser. $\mathrm{Sal})$, las organizaciones sociales locales (OSL) y las organizaciones ambientalistas (OA), y que el análisis biblot simétrico de Gabriel (Gabriel, 1971) reveló como principales para configurar el perfil de los ciudadanos en torno al proyecto que generó el conflicto de Termoeléctrica Castilla.

\subsection{Caracterización de los grupos biplot y confianza}

El análisis de perfilamiento de los actores que contestaron a la encuesta se configura entorno al biplot de la figura 1 . 
Figura 1. Representación factorial del biplot simétrico de confianza

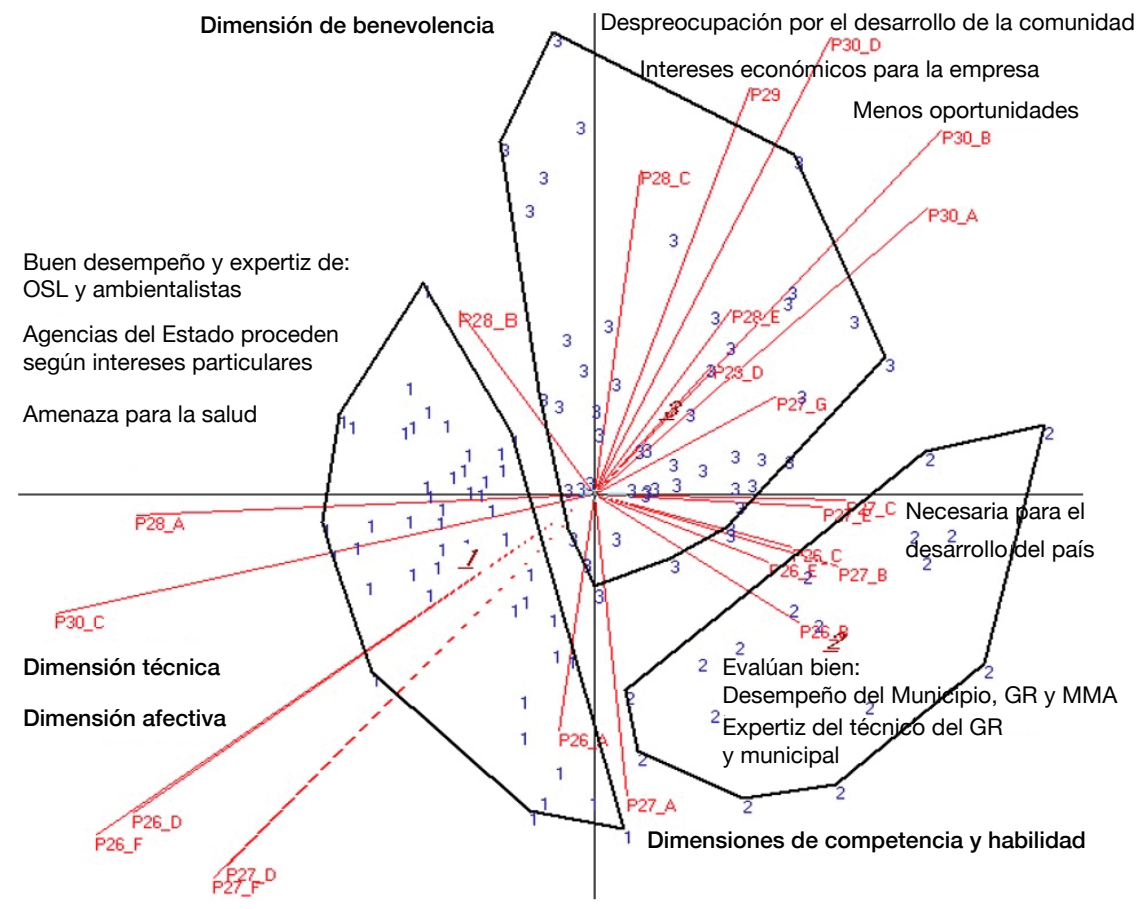

Fuente: elaboración propia.

Para la caracterización de los grupos, se compararon los promedios de cada una de las variables por los grupos obtenidos, con el fin de determinar las variables relevantes para cada grupo y las diferencias promedio estadísticamente significativas. En el anexo 1, se presentan las distribuciones de los promedios estandarizados por grupos. En el análisis, se determinaron tres grupos:

- Grupo 1: constituido por 98 individuos que representan el 31,7\% del total encuestado.

- Grupo 2: conformado por 30 individuos que representan el 9,7\% del total encuestado.

- Grupo 3: establecido por 181 individuos que corresponden al 58,6\% del total encuestado.

A continuación, se describe el perfil y las características de cada grupo.

Grupo 1

El perfil del grupo 1 se ordena en función de las dimensiones de competencia y habilidad, consistencia de comportamientos y benevolencia, donde se 
distinguen las siguientes variables: eficiencia, competencias, preocupación por la salud y el bien común (ver tabla 1). En este grupo, se encuentran aquellos individuos que evaluaron positivamente («bien» o «muy bien») «el desempeño de las organizaciones locales y las organizaciones ambientalistas en el abordaje del conflicto de Termoeléctrica Castilla». En ese mismo sentido, frente a la pregunta de "cómo las organizaciones implicadas han actuado ante el conflicto socioambiental, considerando su experticia, recursos técnicos, financieros, humanos e infraestructura», son las organizaciones sociales locales y las organizaciones ambientalistas las valoradas más positivamente por su actuación. Por el contrario, el rol ante el conflicto de organismos como el Municipio, el Gobierno Regional, el Ministerio del Medio Ambiente y la Secretaría Regional Ministerial de Salud fue evaluado más negativamente («malo» o «muy malo»). Lo anterior es absolutamente coherente con la variable que indaga si «Las agencias del Estado, por lo general, actúan y proceden de acuerdo con sus intereses particulares", donde, mayoritariamente, el grupo estuvo de acuerdo o muy de acuerdo. Finalmente, la variable que releva la idea de que «la puesta en funcionamiento de Castilla sería una amenaza para la salud de las personas» es vista como una cuestión factible, ya que sus observaciones se agrupan mayoritariamente en un "de acuerdo» o en un «muy de acuerdo" ante el enunciado.

En función de las variables que caracterizan a este grupo, se puede sostener que este es un colectivo que toma distancia de la institucionalidad pública, puesto que no deposita tanta confianza en las competencias técnicas y afectivas de las agencias estatales como en las de las organizaciones de base.

Para complementar su perfil, se consideraron las dimensiones de «Identificación e inclusión social» presentes en la encuesta aplicada. De ello, se desprende que, mayoritariamente, este grupo está compuesto por ciudadanos con una cultura cívica proambiental más bien elevada, que se sienten informados y que interactúan con distintos medios (televisiones, periódicos, Internet, radio, etc.), que hablan de política tanto con la familia como con los amigos, que demuestran disposición a sumarse a acciones colectivas contenciosas y la mayoría tienen confianza en las personas que les rodean. Finalmente, es importante destacar que el 93\% de los individuos de este grupo no estaba de acuerdo con «la construcción de la Termoeléctrica Castilla».

\section{Grupo 2}

El perfil del grupo 2 se conforma en oposición a las observaciones referidas para el grupo 1, puesto que las variables relevantes que lo constituyen valoran positivamente el desempeño, la experticia y la eficacia de las agencias estatales y de la empresa. Variable central de este grupo es la dimensión general competencia y habilidad, que posee una capacidad heurística importante para explicar su percepción de confianza.

Así, ante la interrogante de «¿Cómo evaluaría el desempeño en el abordaje del conflicto Termoeléctrica Castilla de las siguientes instancias...?», el Gobierno Regional, el Ministerio del Medio Ambiente y la empresa gestora resultan con evaluaciones positivas. Como se ve, se trata de una situación muy distinta 
a las variables que caracterizaron al grupo 1, donde factores explicativos de la confianza residían en la capacidad y la acción de las organizaciones sociales (ambientalistas). Por otra parte, el Municipio no aparece en ningún caso como actor relevante, en uno u otro sentido, situación consistente con la institucionalidad ambiental, en tanto no le otorga ninguna competencia. Con respecto a la variable que «evalúa la acción de las organizaciones y entidades en el conflicto, tomando en cuenta su experticia y eficacia sobre la base de la disponibilidad de recursos técnicos, financieros, humanos e infraestructura», los ejes que se destacan posicionan nuevamente a las mismas instancias anteriores. Es decir, en estos términos, los factores articuladores de confianza institucional son percibidos positivamente en el grupo 2, para los principales actores definidos por la institucionalidad ambiental en la instalación y puesta en funcionamiento de proyectos de la envergadura de la Termoeléctrica Castilla.

Lo interesante es que estas evaluaciones no guardan una relación directa con la aceptabilidad de las propuestas, entendiendo que los resultados de la encuesta posicionan a menos del $50 \%$ de los individuos, cuyo perfil se distingue en el grupo 2, de acuerdo con la realización del proyecto.

No obstante, que la gran mayoría de los individuos del grupo 2 expresara que «La construcción de la Termoeléctrica Castilla era necesaria para el desarrollo del país y que debió realizarse respetando la normativa ambiental» explica que solo una minoría (un 22\%) declare que «La gestión de Castilla dio cuenta de una empresa transparente». En otro sentido, el perfil del grupo 2 se articula en la precaria confianza hacia los dispositivos comunicacionales, en particular, en los canales de televisión, y siente poca confianza en el futuro del país. Asimismo, otra característica de este grupo es su actitud indiferente a participar en acciones colectivas de carácter contencioso que no involucren directamente sus propios intereses.

\section{Grupo 3}

Finalmente, el grupo 3 está caracterizado por las variables que indagan sobre las oportunidades, la preocupación y la ganancia económica que el proyecto hubiera traído para la región y la comunidad. La mayoría de los ciudadanos se ubican bajo el promedio en estas variables (un 75\%), por lo que el perfil de este grupo se caracteriza por variables que se relevan en torno a las preguntas que interrogan «si se hubiera implementado el proyecto Termoeléctrica Castilla, este pudo haber traído mayores oportunidades»; si «Castilla demostró preocupación por los intereses de la comunidad»; si «desde sus inicios el proyecto pensó en el desarrollo de los habitantes de la región» y si «el proyecto Castilla no pensó solo en la ganancia económica, sino que apostó por el desarrollo de la región».

Como se observa, se trata de apreciaciones muy distintas a las sostenidas en los grupos anteriores, en tanto la composición del grupo se articula en función de la dimensión benevolencia (ver la tabla 1). Las variables que confirman este perfil están dadas por sus prácticas y sus relaciones sociales, y se muestran en desacuerdo con la vinculación a organizaciones políticas. Manifiestan también un abierto rechazo a la participación en cualquier forma de organización colectiva, 
ya sea esta una manifestación social de reclamo u organización social de distinta naturaleza; poseen un débil capital social, donde un porcentaje muy elevado considera que no se puede confiar en las personas; sus hábitos para informarse se reducen a medios tradicionales, es decir, no usan redes sociales ni tampoco Internet, aunque desconfían de la información entregada por la televisión abierta. Respecto al proyecto Termoeléctrica Castilla, en forma contundente, asumen que tendría impactos negativos en el medio ambiente, aun cuando su aceptabilidad a la instalación y al desarrollo de la propuesta es difusa, puesto que perciben también oportunidades económicas en el proyecto. En síntesis, se trata de un conjunto de individuos donde priman valores individualistas, de poca información, con desconfianza social e institucional. En ese sentido, se trata de un grupo con el cual es potencialmente difícil establecer un diálogo social.

Por último, en la figura 2, se muestra cómo se articula la relación entre los tres grupos de actores identificados, de acuerdo con los perfiles establecidos en torno a las dimensiones de la confianza (presentados anteriormente en la tabla 1) y en relación con la mayor o menor disposición de la acción de la ciudadanía respecto al proyecto. Un proyecto cuyos riesgos potenciales para las personas, su entorno natural y cultural, generaron un conflicto socioambiental manifiesto.

Figura 2. Esquema de perfil y variables de la percepción de confianza

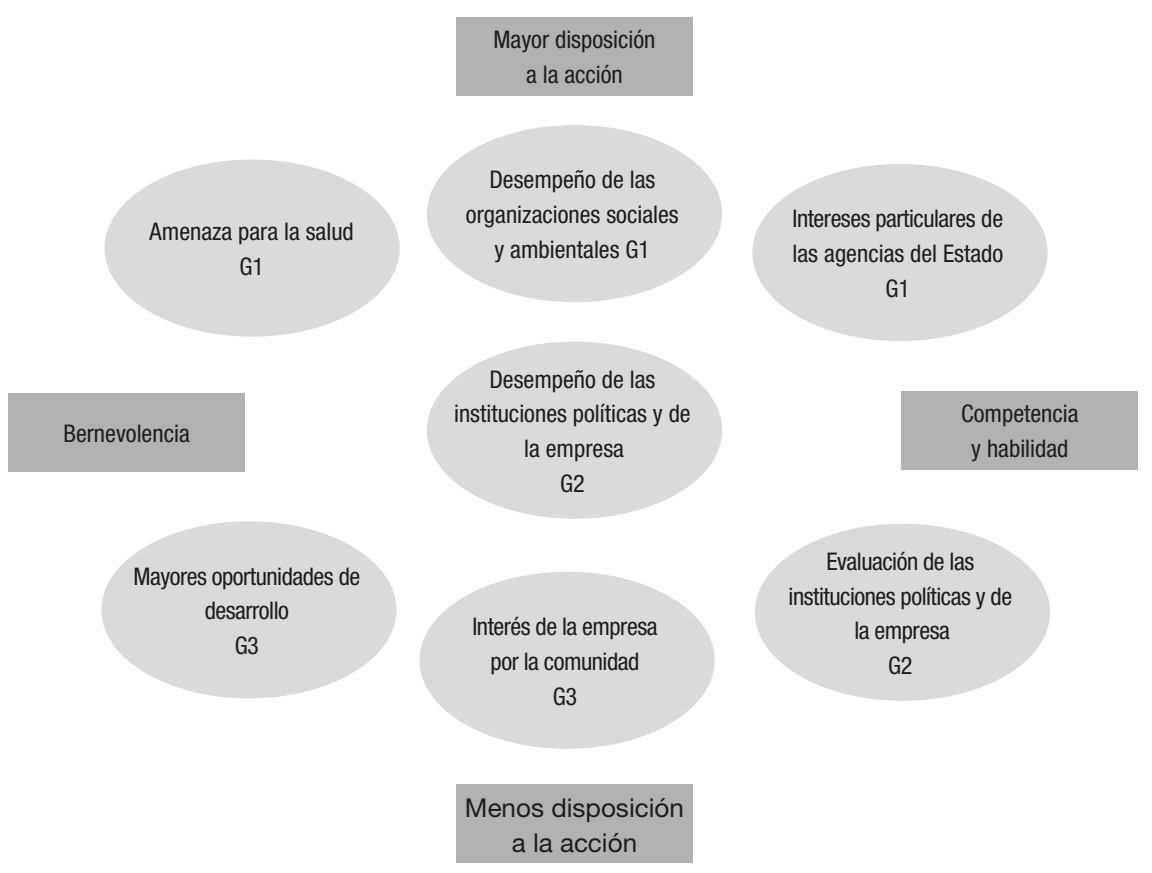

Fuente: elaboración propia. 


\section{Conclusiones}

La cuestión de la confianza en las instituciones públicas y privadas es cada vez más relevante para la gestión de riesgos. En la línea argumentativa expuesta, no solo cobran importancia las constataciones y las mediciones técnicas, en sí complejas, inciertas y debatibles, sino también la consideración de dimensiones sociales (como la confianza), que, según la literatura, se han tornado relevantes para dar cuenta de un mejor manejo de las externalidades traducidas en riesgos y peligros que se evidencian en problemas y conflictos socioambientales que la sociedad genera. En una sociedad moderna como la chilena, que en su seno produce y reproduce una alta conflictividad socioambiental, generada, entre otros muchos ejes, por proyectos de inversión ligados a la producción y a la distribución de energías, estas dinámicas tienden a aumentar debido a la alta demanda de crecimiento económico.

El análisis de clúster basado en los factores (componentes) obtenidos con el biplot simétrico de Gabriel nos permite caracterizar y analizar los tres perfiles diferenciados de individuos que respondieron la encuesta. La relevancia de lo obtenido radica en que nos permite comprender determinadas construcciones y acciones sociales en función de la interrelación social que establecen los individuos con las instancias públicas y privadas ligadas al conflicto de la Termoeléctrica Castilla. Una dimensión social de los riesgos que permite dar cuenta de la mayor o menor aceptación de un proyecto que incide, tanto positiva como negativamente (según los grupos descritos), en las personas, la comunidad en general y, principalmente, en la percepción y sanción de un proyecto con profundas implicaciones para su futuro y su calidad de vida. De fondo, nos permite constatar que, tal como están planteados el problema y la literatura expuesta, no bastan variables técnicas y políticas para decidir si un proyecto es viable o no, sino que la confianza, como reductor de complejidad y cemento social (Luhmann, 1996a), es una variable clave a ser tenida en cuenta para que un proyecto o megaproyecto energético sea viable socialmente.

La experiencia del conflicto de la Termoeléctrica Castilla nos reafirma el argumento que recorre este texto, pues, al existir mermadas relaciones de confianza entre las instituciones públicas y privadas y los actores sociales, las interacciones se tensionan y producen efectos que limitan la gestión de los riesgos evidenciados por los conflictos socioambientales, y los proyectos y los conflictos manifiestos terminan dejando ganadores y perdedores, además de una confianza debilitada, la cual es muy díficil de recomponer (Espluga et al., 2009; Rodríguez y Majluf, 2003 Luhmann, 1996a). Por lo tanto, la confianza como posibilitadora de estabilidad social nos permitiría tener mejores chances para dar cuenta de una sociedad cada vez más contingente, donde casos como el de Castilla nos muestran que las decisiones tomadas tienen más probabilidades de ser frustradas que de tener un final feliz. En este caso, se evidencia el error de prescindir de esta variable fundamental para la gobernanza del riesgo, dado que más de un $90 \%$ de los encuestados perfilados en los tres grupos está en contra de la concreción de un proyecto como Castilla, lo cual podría traer más decepciones que buenos finales para los promotores del proyecto. 
Junto a lo anterior, analíticamente, podemos establecer una nueva distinción de los perfiles resultantes del análisis multivariado, de acuerdo con su mayor o menor disposición para la acción colectiva, categoría emergente que se hace absolutamente relevante y amplía la capacidad heurística de nuestros resultados, sobre todo al vincularla al marco conceptual de las dimensiones que mostraron mayor variabilidad en el análisis biplot, como son competencia y habilidad y benevolencia, a ser tenidas en cuenta en la gestión y en la intervención de estos fenómenos sociales.

En cuanto al modelo teórico y conceptual sobre la percepción de confianza (expuesto en la primera parte del texto), nuestros resultados no sugieren que solo las dimensiones citadas sean las importantes, sino más bien que son las que tienen mayor variabilidad y las destacadas por el modelo de análisis escogido. En otras palabras, son aquellas variables que mayor peso tienen en la configuración de posiciones de los actores del conflicto y que adquieren relevancia en un contexto de desafíos para la institucionalidad pública como el descrito. En este sentido, los bajos niveles de confianza en la actuación de los poderes públicos y de la empresa, mostrados por los grupos 1 y 3 de nuestro análisis, son elementos clave para explicar el conflicto, así como una muestra de las dificultades para encauzarlo. La resolución de este tipo de conflictos socioambientales requeriría de un nuevo diseño institucional que permitiera implicar a estos grupos sociales en diferentes grados de la toma de decisiones sobre el proyecto y sus riesgos. Se evidencia, pues, la conveniencia de nuevas fórmulas que permitan efectuar una gestión más deliberativa de los proyectos tecnológicos, con mecanismos de comunicación multidireccionales y con la inclusión de una pluralidad de visiones en la toma de decisiones, al menos de la población que se percibe afectada por los posibles riesgos.

\section{Agradecimientos}

Este artículo es producto del proyecto Fondecyt 1120554, Las dimensiones sociales, confianza y comunicación de los riesgos ecológicos en conflictos socioambientales por energía en Chile: Los casos de Castilla e HidroAysén, financiado por el Gobierno de Chile.

\section{Referencias bibliográficas}

Aguilar, Cristian y Valenzuela, Ricardo (2010). Crisis energética: Propuestas cientificas en energias renovables no convencionales en Chile entre los años 2000 y 2010. Chile: Universidad de La Frontera. Tesis de licenciatura.

Altieri, Miguel y Rojas, Alejandro (1999). «La tragedia ecológica del milagro neoliberal chileno». Persona y Sociedad, XII (1), 127-141.

Bauman, Zygmunt (2006). Vida líquida. Barcelona: Paidós Ibérica.

Beck, Ulrich (1995). Ecological Enlightment. Nueva Jersey: Humanity Press.

- (2002). La sociedad del riesgo global. Madrid: Siglo XXI.

- (2006). La sociedad del riesgo: Hacia una nueva modernidad. Barcelona: Paidós Ibérica.

- (2008). La sociedad del riesgo mundial: En busca de la seguridad perdida. Barcelona: Paidós. 
Bulloch, Sarah (2013). «Seeking Construct Validity in Interpersonal Trust Research: A Proposal on Linking Theory and Survey Measures». Social Indicators Research [en línea], 113 (3), 1289-1310. <http://dx.doi.org/10.1007/s11205-012-0139-0>.

Camus, Pablo y HayeK, Ernst (1998). Historia ambiental de Chile [en línea]. Santiago de Chile: Ediciones Pontificia Universidad Católica de Chile. <http://dx.doi.org/10.7764/psykhe.22.2.678>.

Espluga, Josep (2004). «Conflictes socioambientals i estudi de la percepció social del risc». Papers, 72, 145-162.

- (2007). "Per una sociología dels riscos tecnològics». Àmbits de Política i Societat, 36, 34-38.

Espluga, Josep y Farré, Jordi (2007). «Más allá de la percepción: La comunicación del riesgo». En: Ferré, Jordi y Fernández, Josep (eds.). Comunicació i risc petroquimic a Tarragona: De les definicions a les pràctiques institucionals. Tarragona: Publicacions de la URV.

Espluga, Josep; Farré, Jordi; Gonzalo, Jan y Prades, Ana (2014) «Factores que inhiben la movilización social: el caso del área petroquímica de Tarragona». Revista Española de Investigaciones Sociológicas, 146, 191-216. <http://dx.doi.org/10.5477/cis/reis.146.191>.

Espluga, Josep; Prades, Ana; Gamero, Nuria y Solá, Rosario (2009). «El papel de la confianza en los conflictos socioambientales». Politica y Sociedad, 46 (1-2), 255-273.

Espluga, Josep; Prades, Ana y Gonzalo, Jan (2010). "Comunicating at the edge: Risk communication processes and structural conflicts in highly industrialized petrochemical areas». Catalan Journal of Communication \& Cultural Studies [en línea], 2 (2), 231-251. <http://dx.doi.org/10.1386/cjcs.2.2.231_1>.

Fiske, Emmett (2000). «Reconceptualizing Environmental Conflict Resolution: The Development Facilitation Approach». Ponencia presentada en la conferencia Alternative Dispute Resolution and Natural Resources: Building Consensus and Resolving Conficts in the Twenty-first-Century. Tucson, Arizona.

Gabriel, Ruben (1971). "The biplot graphic display of matrices with application to principal component analysis». Biometrika [en línea], 58 (3), 453-467. <http://dx.doi.org/10.1093/biomet/58.3.453>.

Giddens, Anthony (1993). Las consecuencias de la modernidad. Madrid: Alianza.

- (1996a). Más allá de la izquierda y la derecha. Madrid: Cátedra.

- (1996b). «Modernidad y autoidentidad». En: Beriain, Josetxo (comp.). Las consecuencias perversas de la modernidad. Barcelona: Anthropos.

Giro País (2011). Qué implica la Termoeléctrica Castilla [en línea]. <http://www. giropais.cl/content/view/1410869/Queimplica-la-Termoelectrica-Castilla.html> [Consulta: 3 mayo 2013].

Luhmann, Niklas (1992). Sociología del riesgo. México: Triana / Universidad Iberoamericana.

- (1996a). Confianza. Barcelona: Anthropos.

- (1996b). «¿Puede la sociedad moderna evitar los peligros ecológicos?». Argumentos, 24, 7-18.

- (2007). La sociedad de la sociedad. México: Herder / Universidad Iberoamericana.

Majluf, Nicolás; Abarca, Nureya y Mingo, Santiago (2003). La confianza en la empresa. Santiago de Chile: Pontificia Universidad Católica de Chile. Departamento de Ingeniería Industrial. Documento de trabajo. 
OCDE (2005). Evaluaciones del desempeño ambiental [en línea]. Santiago de Chile: CEPAL. <http://dx.doi.org/10.1787/9789264189188-13-es>.

Quiroga, Rayen (2001). «La sustentabilidad socioambiental de la emergente economía chilena entre 1974 y 1999: Evidencias y desafíos». En: SADER, Emir (comp.). El ajuste estructural en América Latina: Costos sociales y alternativas. Buenos Aires: CLACSO.

Ricaldi, Teresa (1999). Economía ecológica: Una nueva mirada a la ecología humana. Bolivia: UNESCO/CESU-UMSS.

Rodríguez, Darío y Majluf, Nicolás (2003). La Confianza en Chile: Sus dificultades y posibilidades. Santiago de Chile: Pontificia Universidad Católica de Chile. Departamento de Ingeniería Industrial. Documento de trabajo.

Rodríguez, Hannot (2009). «La confianza pública en las instituciones reguladoras de riesgo: Tres modelos de confianza para tres desafíos del análisis del riesgo». Argumentos de Razón Técnica, 12, 125-153.

Rojas, Alejandro (1995). «El caso de Clayoquot Sound, Canadá: Confrontación, paradigma y diálogo intercultural en la negociación de un conflicto medioambiental». Ambiente y Desarrollo, XI (2), 67-75.

Sabatini, Francisco (1998). "Los conflictos ambientales en Chile: Conclusiones de investigación y perspectivas». Ambiente y Desarrollo, XIV (4), 6-12.

Sabatini, Francisco y Sepúlveda, Claudia (1997). Conflictos ambientales: Entre la globalización y la sociedad civil. Santiago de Chile: CIPMA.

Sepúlveda, Claudia y Geisse, Guillermo (1995). «El caso de Golden Sping: La construcción social de la demanda ambiental entre los habitantes de Compu». Ambiente y Desarrollo, XI (1), 59-66.

Sepúlveda, Claudia y Rojas, Alejandro (2010). «Conflictos ambientales y reforma ambiental en Chile: Una oportunidad desaprovechada». Ambiente y Desarrollo, XXIV (2), 15-23.

VAIRINHOS, Valter (2003). Desarrollo de un sistema para minería de datos basado en los métodos BIPLOT. España: Universidad de Salamanca. Tesis para optar al grado de doctor.

Vallejos-Romero, Arturo; Salvo, Sonia; Garrido, Jaime y Espluga, Josep (2014). La comunicación de riesgos en conflictos socioambientales: Una revisión teórico-conceptual y critica. Mimeo.

Vilches, Carlos (2010). Vilches (UDI) defiende que se instale Termoeléctrica Castilla en zona donde tiene intereses mineros [en línea]. <http://www.elmostrador.cl/noticias/ pais/2010/11/16/vilches-udi-dice-que-dictamen-determoelectrica-castilla-debeser-corregido-y-permitir-instalacion/>_[Consulta: 10 mayo 2013].

VVAA (2006). Informe Pais: Estado del medio ambiente en Chile [en línea]. Santiago de Chile: Universidad de Chile. INAP. <http://dx.doi.org/10.5354/0365-7779.1861.6286>.

VVAA (2010). Escenarios energéticos Chile 2030. "Construyendo escenarios y desafiando paradigmas» [en línea]. <http://www.escenariosenergeticos.cl/wp/wp-content/ uploads/2010/08/Escenarios-Energ\%C3\%A9ticos_Chile-20301.pdf> [Consulta: 10 mayo 2013].

Wynne, Brian (1992). "Risk and Social Learning: Reification to Engagement». En: Krimsky, Sheldon y Golding, Dominic (eds.). Social Theories of Risk. Westport, Londres: Praeger.

- (1996). «Misunderstood Misunderstanding: Social Identities and Public Uptake of Science». En: Lash, Scott; Szerszynski, Bronislaw y Wynne, Brian. Risk, Environment and Modernity. Londres: Sage. 


\section{Anexo 1. Distribuciones de los promedios estandarizados por grupos}

La figura 3 nos muestra el promedio estandarizado (media 0 y desviación estándar 1) por grupos acerca de cómo se distribuye la evaluación que hace la ciudadanía respecto al desempeño que tuvieron las instancias públicas para abordar el conflicto de la Termoeléctrica Castilla.

Figura 3. Evaluación del desempeño de las instancias públicas
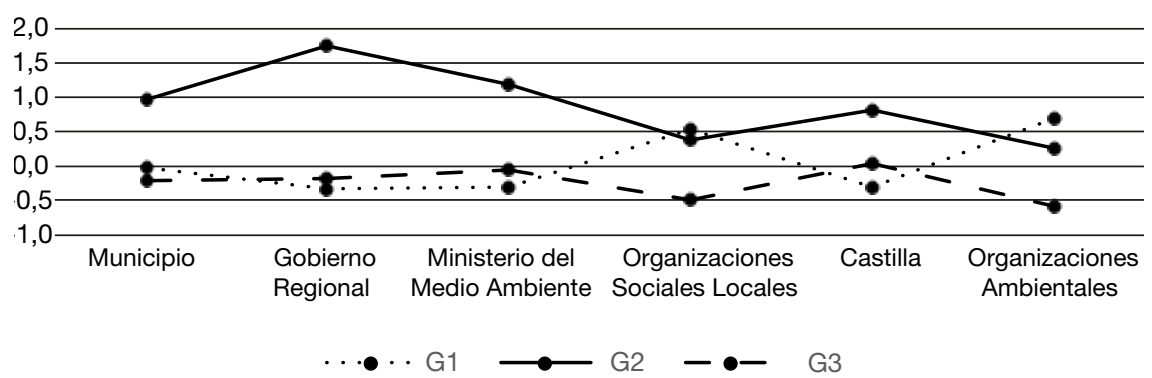

La figura 4 presenta la distribución del promedio estandarizado (media cero y desviación estándar uno) del funcionamiento que tuvieron las instancias públicas y privadas respecto a sus recursos técnicos, financieros, humanos y de infraestructura, variables que la literatura (ver tabla 1) expone como «confianza objetiva».

Figura 4. Funcionamiento de instancias públicas según la confianza objetiva
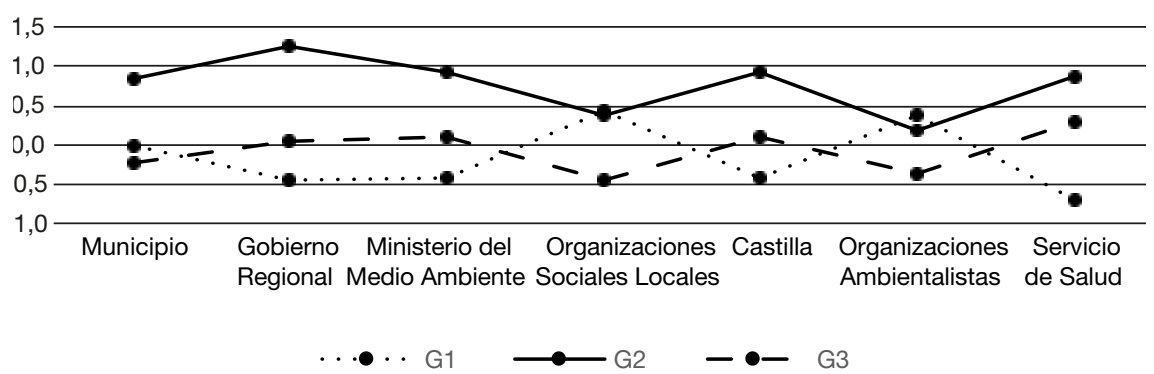

La figura 5 nos muestra el promedio estandarizado (media cero y desviación estándar uno) por grupo respecto del nivel de acuerdo o desacuerdo (escala de 1 a 5; «muy en desacuerdo» a "muy de acuerdo») ante las siguientes interrogantes: F1) si las agencias del Estado actuaron y procedieron de acuerdo con sus intereses particulares; F2) si, ante la conflictividad social, la acción pública demostró una posición única y consistente con sus propósitos; F3) si las prác- 
ticas de las agencias del Estado fueron claras y previsibles; F4) si la empresa Castilla demostró una práctica coherente y consistente con su discurso; F5) si la institucionalidad ambiental en Chile es justa y transparente, y F6) si, ante la idea de mejorar la calidad de vida de la gente, el proyecto Termoeléctrica Castilla iba a traer mayores oportunidades, las mismas o menores.

Figura 5. Desempeño de las instancias públicas y privadas según la confianza subjetiva

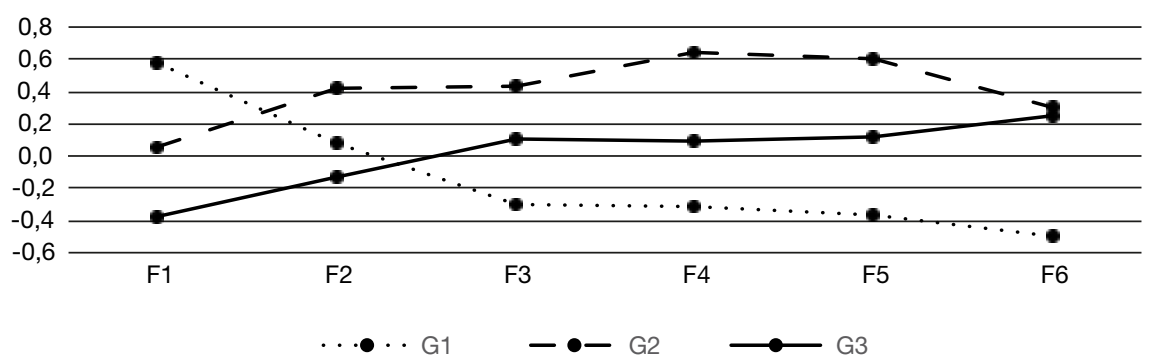

Por último, en la figura 6 , se presenta la distribución del promedio estandarizado (media cero y desviación estándar uno) por grupo respecto del nivel de acuerdo o desacuerdo (escala de 1 a 5 , «muy en desacuerdo» o «muy de acuerdo») con las sentencias siguientes: F1) si «la empresa Castilla demostró preocupación por los intereses de la comunidad»; F2) si «desde sus inicios el Proyecto Termoeléctrica Castilla pensó en el desarrollo de los habitantes de la región»; F3) si «el desarrollo del proyecto Castilla hubiera sido una amenaza para la salud de las personas», y F4) si «el Proyecto Castilla no pensó solo en la ganancia económica de la empresa, sino que apostó también por el desarrollo de la región».

Figura 6. Desempeño de instancias privadas según la confianza subjetiva

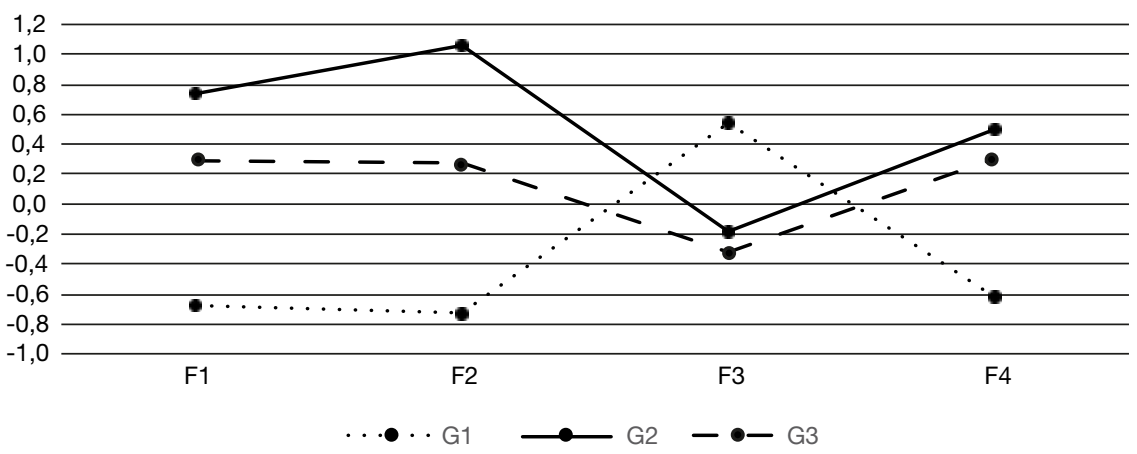

